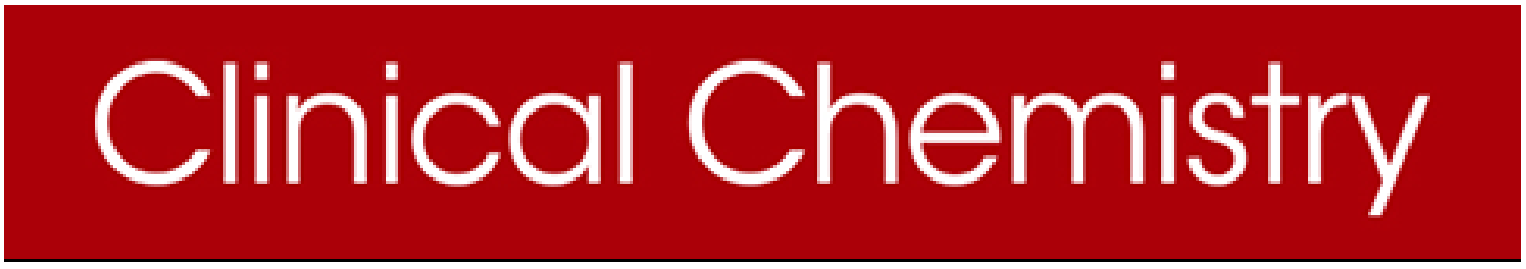

Manuscript Title: The IFCC Clinical Application of Cardiac Biomarkers Committee's Appraisal of the 2020 ESC Guidelines for the Management of Acute Coronary Syndromes in Patients Presenting Without Persistent ST-segment Elevation: Getting Cardiac Troponin Right

Manuscript No: CLINCHEM/2020/332908 [R1]

Manuscript Type: Special Report

Date Submitted by the Author: 14 Dec 2020

Complete List of Authors: Fred S Apple, Paul Collinson, Peter Kavsak, Richard Body, Jordi Ordóñez-Llanos, Amy Saenger, Torbjorn Omland, Ola Hammarsten, and Allan Jaffe

Keywords: 99th percentile; biomarkers; cardiac troponin; myocardial infarction; risk outcomes ; sex-specific reference limits

Dear Reviewer,

Thank you for reviewing this manuscript, please remember the following:

1. The attached manuscript is confidential and should not be circulated or shared with anyone.

2. Authors of this manuscript should never be contacted until after its publication.

3. If you have a conflict of interest regarding this work, contact the editorial office immediately.

4. Be sure to review the authors' potential conflicts of interest by following the "Author Disclosures" link in your reviewer area. Contact the editorial office if you need assistance. 
The IFCC Clinical Application of Cardiac Biomarkers Committee's Appraisal of the 2020 ESC Guidelines for the Management of Acute Coronary Syndromes in Patients Presenting Without Persistent ST-segment Elevation: Getting Cardiac Troponin Right

Fred S. Apple ${ }^{1^{*}}$, Paul O. Collinson ${ }^{2}$,Peter A. Kavsak ${ }^{3}$, Richard Body ${ }^{4}$, Jordi OrdóñezLlanos $^{5}$, Amy K. Saenger ${ }^{1}$, Tobjørn Omland ${ }^{6}$, Ola Hammarsten ${ }^{7}$, Allan S. Jaffe ${ }^{8}$, on behalf of the IFCC Committee Clinical Application of Cardiac Biomarkers

1. Department of Laboratory Medicine and Pathology, Hennepin Healthcare/HCMC, Minneapolis, MN 55415, USA and Department of Laboratory Medicine and Pathology, University of Minnesota, Minneapolis, MN, USA

2. Departments of Clinical Blood Sciences and Cardiology, St George's University Hospitals NHS Foundation Trust and St George's University of London, London, UK

3. Department of Pathology and Molecular Medicine, McMaster University, Hamilton, Ontario, Canada

4. Emergency Department, Central Manchester University Hospitals NHS Foundation Trust, Manchester Academic Health Science Centre, Manchester, UK; Cardiovascular Sciences Research Group, Core Technology Facility, Manchester, UK; and Healthcare Sciences Department, Manchester Metropolitan University, Manchester, UK 
5. Servicio de Bioquímica Clínica, Institut d'Investigacions Biomèdiques Sant Pau, Barcelona, Spain; and Departamento de Bioquímica y Biología Molecular, Universidad Autònoma de Barcelona, Barcelona, Spain

6. Department of Cardiology, Akershus University Hospital, Lørenskog, Norway; and Institute of Clinical Medicine, University of Oslo, Oslo, Norway

7. Department of Clinical Chemistry and Transfusion Medicine, Sahlgrenska University Hospital, Sahlgrenska Academy at the University of Gothenburg, Sweden

8. Departments of Laboratory Medicine and Pathology and Cardiology, Mayo Clinic, Rochester, MN, USA

${ }^{*}$ Corresponding author: Fred S. Apple, Hennepin Healthcare/HCMC, 701 Park Avenue, Clinical Labs P4, Minneapolis, MN 55415, USA; Tele: +1 612 873-3324, Fax: +1 612 904-4229, E-mail: apple004@umn.edu 
Disclosures:

Dr Apple: Board of Directors, HyTest Ltd; Associate Editor, Clinical Chemistry; Advisory Boards, Instrumentation Laboratory, Siemens Healthcare, Osler Diagnostics, Qorvo; Honorarium for Speaking at Industry Conferences, Siemens Healthcare, Abbott Diagnostics; PI on Industry Funded Grants (non-salaried) through research institute (HHRI), Abbott Diagnostics, Abbott POC, BD, Beckman Coulter, Ortho-Clinical Diagnostics, Roche Diagnostics, Siemens Healthcare, ET Healthcare, Quidel;

Dr. Kavsak: grants/reagents/consultant/advisor/ honoraria, Abbott Laboratories, Abbott Point of Care, Beckman Coulter, Ortho

Clinical Diagnostics, Quidel, Randox Laboratories, Roche Diagnostics, Siemens Healthcare Diagnostics; McMaster University has filed patents with Dr. Kavsak listed as an inventor in acute cardiovascular biomarker field;

Dr. Jaffe: consultant, Beckman, Abbott, Siemens, Roche, Radiometer, ET Healthcare, Sphingotec, Amgen, Novartis

Dr. Ordonez-Llanos - none.

Dr. Collinson: Associate Editor, Journal Applied Laboratory Medicine;

Dr. Saenger: Advisory Board, Radiometer;

Dr.Body: Consultant, LumiraDx, Beckman, Siemens; Donation of reagents for research and or grant, Roche, Abbott POC, Beckman;

Dr. Hammarsten: None;

Dr. Omland: Consultant or Advisory Role, Abbott Diagnostics, Roche Diagnostics, CardiNor; Honoraria, Abbott Diagnostics, Roche Diagnostics; Research Funding, Abbott Diagnostics, Roche Diagnostics, SomaLogic, Novartis, all to institution. 
The Committee on the Clinical Application of Cardiac Biomarkers (C-CB) of the International Federation of Clinical Chemistry (IFCC) represents international groups from laboratory medicine, cardiology and emergency medicine involved with providing global educational guidance pertaining to the analytical and clinical applications of cardiac biomarkers. For that reason, most of the members are involved with national and international studies and trials pertaining to high sensitivity (hs)-cTnl and hs-cTnT (1-3). Although the recently published '2020 European Society of Cardiology (ESC) guidelines for the management of acute coronary syndromes in patients presenting without persistent ST-segment elevation' do somethings very well, this special report was developed to delineate our specific concerns regarding the guidance for the use of hs-cTn (4). Guidelines should be based on a systematic review of the literature, assessment of quality and bias of the evidence, and recommendations should be made with input from a multi-disciplinary group with active participation from cardiology, laboratory medicine and emergency medicine. Instead, the section on hs-cTn is overly focused on a single research consortium which subsumes $>50 \%$ of all references. Thus, it fails in our estimation to accommodate certain areas of practice that are important on a global scale, not just in Europe. The topics we address in this article would have been identified if the guidelines had been developed with more active participation from Laboratory Medicine and vetted more extensively. Many of the concerns being expressed in the current document are relevant to the international laboratory medicine community. This gap in the review process is surprising given the large number of analytical and clinical biomarker experts in Europe and the presence of a designated 
ESC Biomarker Committee which contains both laboratorians and clinicians (5).

\section{Universal Definition of Myocardial Infarction (2018)}

The Universal Definition of Myocardial Infarction (2018) (므) endorses the use of sexspecific $99^{\text {th }}$ percentile upper reference limits (URLs) for hs-cTn assays while acknowledging differences between assays. In contrast, the 2020 ESC guidelines state, "the use of uniform cut off concentrations should remain the standard of care in the early diagnosis of MI." This ignores the advocacy of the 2018 Universal Definition ()ㅡ which was endorsed by the ESC, as well as guidelines from the IFCC-CB and the Academy of the American Association for Clinical Chemistry (AACC) (므).

The ESC justification for using uniform hs-cTn cut-offs is based on the concept that other confounders like age, renal dysfunction or time from chest pain onset also might also need to be considered to optimize cut-off values in patients presenting with symptoms suggestive of ischemia. The ESC guidelines do acknowledge sex as a confounder (7), but state, "Until information technology tools that allow the incorporation of the effect of all four variables are available, the use of uniform cut-off concentrations should remain the standard of care in the early diagnosis of Ml". We disagree. It is predicated predominantly on findings using a uniform URL for hs-cTnT and fails to recognize the multiple other studies, including hs-cTnT, that provide data suggesting the benefit of such an approach ( $\underline{7-12})$. It also is in contrast to conventions in the field of laboratory medicine that advocates for appropriate and statistically based URLS by sex ()ㅡ, when as in this situation all assays manifest an analytical difference. Indeed multiple issues influence cTn values. Most provide a continuum of effects depending on the magnitude of the influence, as reflected in renal disease for example (13). 
Correcting for all of them would be extremely complex. That fact however should not keep us from correcting the problems associated with the underdiagnosis and under treatment women with myocardial injury and infarction (14). Furthermore, the clinical studies and trials used to validate the shorter triage periods work in large part because they presage eventual adherence to the Universal Definition criteria which is most often used as the gold standard. That fact is not as clear as it might be in the ESC guidelines.

The Universal Definition identifies patients who are "late presenters" as a separate group and warns that they may not manifest significant serial cTn changes over a short time because the downslope of the time-concentration cTn curve is slower than the upslope. It further notes the high degree of variability in the kinetics of hs-cTn in those with ischemic heart disease with many patients reaching peak hs-cTn values early after the onset of symptoms and presentation. The ESC guidelines fail to describe this important group. In our opinion, clinicians need to be sensitive to these patients who have increased cTn concentrations that do not change substantially over an hour or two because they are on the downslope of curve. They deserve additional sampling to ensure they are not simply presenting later after the onset of MI. This terribly important caveat was highlighted initially by the SWEDEHEART Group who reported that $26 \%$ of patients with $\mathrm{MI}$ might not manifest a changing pattern mostly for this reason (15).

\section{Early presenters and kinetics}

The ESC guidelines state "In patients with MI, levels of cardiac troponin rise rapidly (i.e. usually within $1 \mathrm{~h}$ from symptom onset if using high-sensitivity assays) after symptom onset". This statement could be prone to misinterpretation. To detect a change in cTn 
within the reference interval is certainly improved using hs-cTn assays but may be limited for time points close to the index event. There is concern about the use of these rapid diagnostic algorithms for patients presenting $<3$ hours $(\underline{16})$ that is acknowledged

for the single sample, but not for the one-hour rule out. We would argue that this caveat deserves additional emphasis. The issue can be easily missed, because findings from studies using both hs-cTnl and hs-cTnT plotted against time from onset of NSTEMI index events demonstrates that the median time from symptom onset to obtaining the first draw is often $>3$ hours. In addition, the time to first increase above sex-specific URLs is also $>3$ hours, and women are less likely than men to cross those URLs in the baseline sample (16-18). Thus, clinicians may not be sensitive to the fact that early presenters can be a problem.

\section{Overdiagnosis of myocardial injury and infarction}

The ESC also states, "Data from large multicenter studies have consistently shown that hs-cTn increases diagnostic accuracy for $\mathrm{Ml}$ at presentation as compared with conventional assays". This statement is only true when transitioning from the very analytically, insensitive $4^{\text {th }}$ gen cTnT assay to the hs-cTnT assay where a change in MI rates from $22 \%$ to $36 \%$ has been reported (19). This substantial increase is not observed with a transition from an analytical sensitive contemporary cTnl to a hs-cTnl assay. This has been reported for both Abbott (20) and Siemens (21) assays. This misconception has fueled clinicians concerns about an anticipated, large numbers of increased cTn concentrations above URLs which would overwhelm their practice. This outcome is not likely when transitioning from good contemporary cTnl assays. This is even more the case in more heterogeneous patient populations with poorer healthcare, 
as often found in inner city USA emergency departments, which also exist in some areas of Europe. hs-cTn assays do not necessarily translate into higher clinical sensitivities at presentation compared to contemporary assays in ED populations of patients presenting with diverse pathophysiologies for myocardial injury and more type 2 Mls (20).

\section{Conventional vs. high-sensitivity assays}

The most glaring, potentially confusing information is presented in figure 2 of the ESC document. The left panel depicts conventional assays and visualizes concepts from the 1990s. The numbers appear to come from the $4^{\text {th }}$ generation cTnT assay, an analytically insensitive assay that could not distinguish between the $99^{\text {th }}$ percentile URL and the assay's limit of detection (LoD). In fact, many conventional cTnl assays had good analytics as outlined on the IFCC C-CB website (22). The right panel represents a schematic predominately also predicated on the Roche hs-cTnT assay. It does not represent the $99^{\text {th }}$ percentile URLs for hs-cTnl assays, as reported in tables on the IFCC C-CB website (23). The 'red zone' for pathological disease begins at $>20 \mathrm{ng} / \mathrm{L}$, which is substantially less than the overall and sex-specific URLs for many hs-cTnl assays. For example, $99^{\text {th }}$ percentile URLs for the Siemens VISTA and Abbott ARCHITECT hs-cTnl assays (both CE Marked) are: VISTA: overall 59ng/L, female $54 \mathrm{ng} / \mathrm{L}$ and male $79 \mathrm{ng} / \mathrm{L}$; ARCHITECT: overall $26 \mathrm{ng} / \mathrm{L}$, female $16 \mathrm{ng} / \mathrm{L}$ and male $34 \mathrm{ng} / \mathrm{L}$. This misrepresentation will no doubt cause confusion for clinical laboratories and clinicians who attempt to implement these assays based on the ESC guidelines.

\section{Sample types}


The ESC guideline fails to sensitize practitioners to the fact that indicates that cTn URLS are assay and sample type dependent. URLs demonstrate differences in hs-cTn concentrations between serum, lithium heparin plasma, and EDTA plasma $(\underline{3}, \underline{23})$. Laboratories should utilize the same specimen type used in clinical studies to generate the data and algorithms for specific sample types to avoid potential misclassification.

\section{High sensitivity vs. point of care cardiac troponin testing}

The ESC guidelines acknowledge that point-of-care (POC) assays have not been as thoroughly evaluated as automated central laboratory assays. They then proceed to advocate for their use based on clinical performance criteria alone which ignores several analytical issues. This is highlighted by the following statement, "The first hscTnI POCTs have recently been shown to provide comparable performance characteristics to that of central laboratory hs-cTn I/T assays". This statement only applies to clinical studies where specimens were bio-banked and plasma specimens, not whole blood was analyzed by research technologists. For many POC assays, the jump from plasma to whole blood is a substantial challenge. In addition, it does not address analytical performance aspects that might be influenced when non-laboratory staff conduct POC testing (24). We concur with the recent NICE guidelines that until a POC device is appropriately validated using whole blood it should not be designated as hs-cTn POC assay (드). We would suggest the ESC position should as well.

\section{Other markers than cardiac troponin}

The guidelines state, "Myosin-binding protein $\mathrm{C}(\mathrm{cMyC})$ is more abundant than $\mathrm{cTn}$ and may therefore provide value as an alternative to, or in combination with, cardiac 
troponin". This recommendation is concerning as study data are extremely limited, originate predominately from one or two research laboratories using partially automated research assays which take hours to report results, and are poorly biologically and analytically validated outside of acute coronary syndrome patients. In the published literature, $\mathrm{cMyC}$ measured with a so-called high-sensitivity immunoassay, the sensitivity and specificity for Ml diagnosis was comparable to that of hs-cTnT or hs-cTnl (르). Not only is it not ready for clinical use, it also does not have any regulatory approval for clinical use at present. Further, guidelines state, "Routine use of copeptin as additional biomarker for early rule-out of $\mathrm{MI}$ is recommended in increasingly uncommon settings where hs-cTn assays are not available. Copeptin does not have relevant added value for institutions using one of the well-validated hs-cTn-based rapid protocols in the early diagnosis of Ml". We find this recommendation confusing. Both the Universal Definition and the ESC guidelines advocate for hs-cTn assays. Why incur the regulatory burden, cost and added logistics of implementation of an additional test that adds no real value in conjunction with hs-cTn and is not well validated for detection of patients with type 2 $\mathrm{Ml}$ and myocardial injury. Further, recent studies have concluded that compared to several "rapid" rule-out diagnostic strategies the combined use of copeptin and hs-cTn was not as safe (27) and that copeptin does not demonstrate early release kinetics as do hs-cTnl and hs-cTnT after experimental coronary balloon occlusion (28).

\section{Rule-in and rule-out algorithms}

ESC guidelines address rapid 'rule-in' and 'rule-out' algorithms noting, "This seems to substantially reduce the delay to diagnosis, translating into shorter stays in the emergency department and lower costs". One novel aspect of the guidelines is the 
focus on 0/2-hour algorithms which are more robust in regard to analytical variability than the $0 / 1$-hour algorithms. It is important to note that many of these algorithms have been developed from chest pain only populations for Roche hs-cTnT and Abbott hscTnl assays. The Ortho VITROS, LSI Pathfast and Quidel Triage True assays have only one publication for algorithms (Singulex closed operations in 2019), and none that have evaluated the 'new' 0/1h algorithm that was presented in the ESC document. Despite that, cut-off values which are assay-specific are presented in the guidelines table 5. Except for hs-cTnT, this table needs to be updated for the majority of hs-cTnl assays where in some instances there is a substantial literature. For example, there is peer reviewed literature for the Abbott and Siemens hs-cTnl assays that suggest alternative values. Abbott hs-cTnl algorithms have used $<2 \mathrm{ng} / \mathrm{L}$ and $<5 \mathrm{ng} / \mathrm{L}$ to rule-out Ml predicated on a single sample (29-31). This has also been reported for the Siemens' assays $(\underline{32,33})$. However, the ESC has listed the rule-out cutoff for the Abbott assay as $<4 \mathrm{ng} / \mathrm{L}$. In previous publications, the rule-in cutoff was listed as $\geq 52 \mathrm{ng} / \mathrm{L}$, but now it is $\geq 64 \mathrm{ng} / \mathrm{L}$. Data that supports significant analytical variability is present throughout the low range of hs-cTn assays but has not been adequately addressed regarding how that affects clinical decisions $(\underline{34,35})$. Numerical cutoffs matter as different controls should be used if one is using $<2 \mathrm{ng} / \mathrm{L}$ versus $<4 \mathrm{ng} / \mathrm{L}$. We have checked values that the guideline has published, and note numerous discrepancies.

The High-STEACS group has published widely on hs-cTn algorithms with particular regard to a single test rule-out using a $5 \mathrm{ng} / \mathrm{L}$ threshold (⒈36) along with the USA

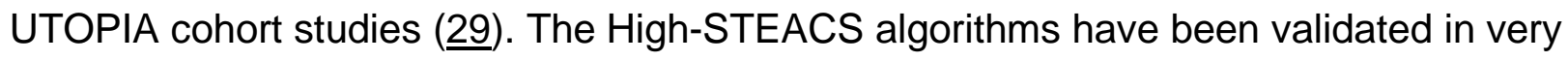
large observational studies and in a large stepped wedge randomized controlled trial 
(37). For those who currently follow those algorithms in practice, the omission appears notable. The same cut off value seems appropriate for the Siemens assays (므).

Both accelerated (01/hour and 0/2 hour) and 0/3hour algorithms are recommended by the ESC for early rule out of MI. It needs to be noted that these are different types of protocols. The accelerated algorithms are based on hs-cTn concentrations at presentation and absolute changes within the first 1 or 2 hours. They are based on the ability to predict that the patient will rule in or out for Ml according to the Universal Definition of Ml on further testing. The guidelines state, "It is recommended to use the 0 $\mathrm{h} / 1 \mathrm{~h}$ algorithm (best option, blood draw at $0 \mathrm{~h}$ and $1 \mathrm{~h}$ ) or the $0 \mathrm{~h} / 2 \mathrm{~h}$ algorithm (second-best option, blood draw at $0 \mathrm{~h}$ and $2 \mathrm{~h}$ ) (ESC Figure 3)." However, the 0/3-hour HighSTEACS pathway is also recommended and is an alternative to the multiple threshold pathways that are designated as 'preferred'. Are there data that it is superior, which should be the criteria employed in guidelines? Based on our own experiences in daily practice with cardiac biomarkers and MI diagnosis, the facts are that baseline specimens typically are a median of about $3 \mathrm{~h}$ from onset of symptoms and the timing of a second draw worldwide is rarely at $1 \mathrm{~h}$. Thus, many of these patients are evaluated closer to 3-4 hours than at 1 or 2 hours. Thus, is it clear that the change criteria will work equivalently in very early presenters (say at 1 or 2 hours) or with the "late presenters." Given these concerns, should the early 0/1-hour protocols be preferred?

No consideration was given to the value of clinical decision aids and risk scores (338). Emergency physicians must take many multiple factors into account when assessing their patients, including the ECG and patient history. Although the ESC guidelines do recommend clinical evaluation, for some who are less experienced in 
cardiology, 'troponin-only' algorithms are used and this is potentially dangerous.

Decision aids force a clinical component on the process. Decision aids are widely used in clinical practice and several have been subjected to randomized controlled trials (HEART impact study; EDACS v TIMI; ADAPT; MACS trial). Their omission from the guideline will appear notable to practicing emergency physicians. This extents to the situation with unstable angina as well. Some clinicians will likely rely on hs-cTn rather than clinical decision making, unless prompted by clinical risk aids so they can make subjective judgements about how to manage patients with ECG changes (e.g. ST depression or T wave inversion) despite normal cTn concentrations.

\section{The path forward}

An international randomized control trial should be carried out addressing both the 0/1 hour and 0/2 hour algorithms for the central laboratory and whole blood POC hs-cTn assays; with events adjudicated by the Universal Definition of Ml using a 0/3 hour protocol. Special attention would be given to both "early" and "late" presenters. This would provide an evidence based option. Such a trial could include novel tools such as

the machine learning myocardial-ischemic-injury-index $\left(\mathrm{Ml}^{3}\right)(\underline{39,40})$. These tools could provide individualized risk that incorporates age, sex, and single and serially paired hscTn results. It could probe the data for the optimal magnitude and rate of change in cTn as a risk estimator for Ml. It could also probe whether fixed concentration thresholds, fixed absolute or percentage changes in concentration, or the mandating specific timepoints for serial testing are necessary.

\section{Summary}


Our IFCC C-CB evidence-based appraisal address numerous concerns about the use of hs-cTn testing in diagnostics that the ESC guidelines are deficient in. Some appear to contradict the ESC endorsed Fourth Universal Definition of Myocardial Infarction as well as Laboratory Medicine practice guidelines. 


\section{References}

1. Apple FS, Collinson PO, and for the IFCC Task Force on Clinical Applications of cardiac Biomarkers: analytical characteristics of high-sensitivity cardiac troponin assays Clin Chem 2012:58:54-61.

2. Apple FS, Jaffe AS, Collinson P, Mockel M, Ordonez-Llanos J, Lindahl B, et al., on behalf of the IFCC Task Force on Clinical Applications of Cardiac BioMarkers. IFCC educational materials on selected analytical and clinical applications of high-sensitivity cardiac troponin assays. Clin Biochem 2015: 2015;48:201-3.

3. Wu AHB, Christenson RH, Greene DN, Jaffe AS, Kavsak PA, Ordonez-Llanos J, Apple FS. Clinical Laboratory Practice Recommendations for the Use of Cardiac Troponin in Acute Coronary Syndrome: Expert Opinion from the Academy of the American Association for Clinical Chemistry and the Task Force on Clinical Applications of Cardiac Bio-Markers of the International Federation of Clinical Chemistry and Laboratory Medicine. Clin Chem 2018;64:645-55.

4. Collet JP, Thiele H, Barbato E, Barthélémy O, Bauersachs J, Bhatt DL, et al, for the ESC Scientific Document Group. 2020 ESC Guidelines for the management of acute coronary syndromes in patients presenting without persistent STsegment elevation. Eur Heart J 2020 Aug 29:ehaa575.

5. Thygesen K, Mair J, Giannitsis E, Mueller C, Lindahl B, Blankenberg S, et al, for the Study Group on Biomarkers in Cardiology of the ESC Working Group on Acute Cardiac Care. How to use high-sensitivity cardiac troponins in acute cardiac care. Eur Heart J 2012; 33:2252-7. 
6. Thygesen K, Alpert JS, Jaffe AS, Chaitman BR, Bax JJ, Morrow, DA, et al. Fourth universal definition of myocardial infarction (2018). Eur Heart $J$ 2018; 40:237-69.

7. Lee KK, Ferry A, Anand A, Strachan F, Chapman AR, Kimenai DM, et al. Highsensitivity troponin with sex-specific thresholds in suspected acute coronary syndrome. J Am Coll Cardiol. 2019;74:2032-43.

8. Apple FS, Wu AHB, Sandoval Y, Sexter A, Love SA, Myers G, Schulz K, Christenson $\mathrm{RH}$. Sex-specific $99^{\text {th }}$ percentile upper reference limits for high sensitivity cardiac troponin assays derived using a universal sample bank. Clin Chem 2020;66:434-44.

9. Shah A, Griffiths M, Lee KK, McAllister MA, Hunter AL, Cruikshank A, Reid A, Stoddart M, Strachan F, Walker S, Collinson PO, Apple FS, Gray AJ, Fox KAA, Newby DE, Mills NL. High-sensitivity cardiac troponin and the under diagnosis of myocardial infarction in women. Brit Med J 2015; 350:7873.

10. Eggers KM, Lindahl B. Impact of sex on cardiac troponin concentrations-a critical appraisal. Clin Chem 2017;63:1457-64.

11. Cullen L, Greenslade JH, Carlton EW, Than M, Pickering JW, Ho A, et al, Sexspecific versus overall cut points for a high sensitivity troponin I assay in predicting 1-year outcomes in emergency patients presenting with chest pain. Heart 2016; 102:308-12.

12. Kimenoi DM, Appelman Y, den Ruijter HM, Shah ASV, Mills NL, Meex SJR. Ten years of high-sensitivity troponin testing: impact on the diagnosis of myocardial infarction. Clin Chem 2020;doi:10.1093/clinchem/hvaa272. 
13. Gunsolus I, Sandoval Y, Smith SW,. Sexter A, Schulz K, Herzog CA, Apple FS. Renal dysfunction influences diagnostics and prognostics of high-sensitivity troponin. J Am Soc Nephrol 2017;29: 636-643.

14. Jaffe AS, Hayes SN. It will take more than better diagnostics to improve the care of women with ACS. J Amer Coll Card 2019; 74: 2044 - 6.

15. Bjurman C, Larsson M, Johanson P. Small changes in troponin T levels are common in patients with non-ST-segment elevation myocardial infarction and are linked to higher mortality. J Am Coll Cardiol 2013; 62: 1231-8.

16. Mueller-Hennessen M, Lindahl B, Giannitsis E, Biener M, Vafaie M, deFilippi CR, et al. Diagnostic and prognostic implications using age- and gender-specific cutoffs for high-sensitivity cardiac troponin T — Sub-analysis from the TRAPID-AMI study. Internat J Cardiol 2016;209:26-33.

17. Sandoval Y, Chapman AR, Mills NL, Than M, Pickering JW, Worster A, Kavsak PA, Apple FS. Sex-specific kinetics of high-sensitivity cardiac troponin I and T following symptom onset and early presentation in non-ST-segment elevation myocardial infarction. Clin Chem.2020; 10.1093/clinchem/hvaa263.

18. Pickering JW, Young JM, George PM, Pemberton CJ, Watson A, Aldos SJ, et al. Early kinetic profiles of troponin I and T measured by high sensitivity assays in patients with myocardial infarction. Clin Chim Acta 2020; In press.

19. Reichlin T, Twerenbold R, Reiter M, Steuer S, Bassetti S, Balmelli C, et al. Introduction of high-sensitivity troponin assays: impact on myocardial infarction incidence and prognosis. Am J Med. 2012;125:1205-13. 
20. Sandoval Y, Smith SW, Thordsen SE, Bruen CA, Carlson MD, Dodd KW, et al. Diagnostic performance of high sensitivity compared to contemporary cardiac troponin I for the diagnosis of acute myocardial infarction. Clin Chem 2017;63:1594-1604.

21. He S, Yang HS, Shemesh A, Li J, Xie,T, Apple FS, Williams J, Zhao Z, Steel P. No increase in the incidence of cardiac troponin I concentration above the 99th percentile by Siemens Centaur high-sensitivity compared to the contemporary assay. Clin Biochem 2020; doi:.

22. https://www.ifcc.org/ifcc-education-division/emd-committees/committee-onclinical-applications-of-cardiac-bio-markers-c-cb/; accessed November 29, 2020.

23. Kavsak PA, Roy C, Malinowski P, Clark L, Lamers S, Bamford K, et al. Sample matrix and high-sensitivity cardiac troponin I assays. Clin Chem Lab Med. $2019 ; 57: 745-51$.

24. Apple FS, Fantz CR, Collinson PO. Implementation of high-sensitivity and point of care cardiac troponin assays into practice: some different thoughts . Clin Chem 2020; In press.

25. Diagnostics guidance [DG40] Diagnostics Assessment Committee National Institute for Health and Care Excellence. High-sensitivity troponin tests for the early rule out of NSTEMI. NICE 26 August 2020; https://www.nice.org.uk/guidance/DG40. 
26. Marjot J, Liebetrau C, Goodson RJ, Kaier T, Weber E, Heseltine P, Marber MS. The development and application of a high-sensitivity immunoassay for cardiac myosin-binding protein C. TransI Res. 2016; 170:17-25.e5.

27. Wildi K, Boeddinghaus J, Nestelberger T, Twerenbold R, Badertscher P, Wussler et al. Comparison of fourteen rule-out strategies for acute myocardial infarction. Int J Cardiol 2019; 283:41-7.

28. Amadottir A, Pedersen S, Hasselbalch RB, Goetz JP, Friis-Hansen LJ, BlochMunster AM, et al. Temporal release of high-sensitivity cardiac troponin T and I and copeptin after brief induced coronary artery balloon occlusion in humans. Circ 2020; doi:10.1161/circulationaha.120.046574.

29. Sandoval Y, Smith SW, Love SA, Sexter A, Schulz K, Apple FS. Single highsensitivity cardiac troponin I to rule out myocardial infarction. Am J Med 2017;130:1076-83.

30. Neumann JT, Twerenbold R, Ojeda F, Sörensen NA, Chapman AR, Shah ASV, et al. Application of high sensitivity cardiac troponin to rule out myocardial infarction. N Engl J Med. 2019;380:2529-40.

31. Shah ASV, Anand A, Strachan FE, Ferry AV, Lee KK, Chapman AR, et al, on behalf of the High-STEACS Investigators High-sensitivity troponin in patients with suspected acute coronary syndrome. Lancet 2018;392:919-28.

32. Sandoval Y, Nowak R, deFilippi CR, Christenson RH, Peacock WF McCord J, et al.. Myocardial infarction risk stratification with a single measurement of highsensitivity troponin I. J Am Coll Cardiol 2019;74:271-282. 
33. Body R, Morris N, Collinson P. Single test rule out of acute myocardial infarction using the limit of detection of a new high-sensitivity troponin I assay. Clin Biochem 2020;78:4-9.

34. Wu AHB, Kavsak PA, Akre KM, Christenson RH, Apple FS, Peacock WF, et al. Lot to lot variation for commercial high sensitivity cardiac troponin: can we realistically report down to the assay's limit of detection. Clin Chem 2020; doi:10.1093/clinchem/hvaa160.

35. Kavsak PA, Don-Wauchope AC, Hill SA, Worster A. Acceptable analytical variation may exceed high-sensitivity cardiac troponin I cutoffs in early rule-out and rule-in acute myocardial infarction algorithms. Clin Chem. 2016;62:887-9.

36. Chapman AR, Hesse K, Andrews J, Lee KK, Anand A, Shah ASV, et al. Highsensitivity cardiac troponin I and clinical risk scores in patients with suspected acute coronary syndrome. Circulation 2018;138:1654-65.

37. Anand A, Lee KK, Chapman AR, et al. High-sensitivity cardiac troponin on presentation to rule out myocardial infarction: a stepped-wedge cluster randomized controlled trial. medRxiv 2020:2020.09.06.20189308. doi: 10.1101/2020.09.06.20189308.

38. Body R, Morris N, Reynard C, Collinson PO. Comparison of four decision aids for the early diagnosis of acute coronary syndromes in the emergency department. Emerg Med J 2019;doi:10.10.1136/energmed-2019-208898.

39. Than MP, Pickering JW, Sandoval Y, Shah ASV, Tsanas A, Apple FS, et al. , on behalf of the MI3 collaborative. Machine learning to predict the likelihood of acute myocardial infarction. Circulation 2019; 140: 899-909. 
40. Neumann JT; Twerenbold R; Ojeda F; Sorensen NA; Chapman AR; Shah ASV; et al. Application of high-sensitivity troponin in suspected myocardial infarction. New Engl J Med 2019;380:2529-40. 